\title{
LA REHABILITACIÓN DEL CENTRO HISTÓRICO DE MADRID
}

\author{
(THE REHABILITATION OF THE HISTORICAL CENTRE OF MADRID)
}

Sigfrido Herráez, Concejal de la Vivienda y Rehabilitación Urbana. Presidente de la E. M. V.

ESPAÑA

Fecha de recepcion: 3-XI1-99

\section{RESUMEN}

El centro histórico de Madrid, debido a su gran extensión, más de 320 heciáreas, presentaba zonas con elevado nivel de deterioro arquitectónico, urbanistico, social y medioambiental, existiendo barrios con gran marginalidad que son refugio de la población inmigrante

Ante el continuo avance del deterioro urbano del centro histórico, las tres administraciones (Estatal, Regional y Local) firmaron varios convenios para la Rehabilitación del Patrimonio Residencial y Urbano, los cuales, junto con los Fondos de Cohesión y los proyectos URBAN para Itinerarios de Cines y Teatros, ambos financiados por la Unión Europea, pretenden regenerar la trama urbana. La coordinación y gestión está encomendada a la Empresa Municipal de la Vivienda.

La fórmula elegida para intervenir en el centro histórico ha sido la declaración de Áreas de Rehabilitación Preferente, centradas en las plazas o calles principales de cada barrio.

Otra fórmula para intervenir ha sido la rehabilitación de las vias urbanas de Itinerarios de Cines y Teatros acogidos a los proyectos URBAN, cuya trama se conecta con las áreas de rehabilitación conteniendo unas calles que son focos de prostitución y marginación social. Los Fondos de Cohesion para mejora del medio ambiente urbano de Lavapiés pretenden adecuar este barrio a las nuevas necesidades de su población.

Las intervenciones tienen como objetivo la renovación de las infraestructuras, mejora del diseño de sus calles, accesibilidad, mejora del medio ambiente, rehabilitación de inmuebles para una mejor calidad de vida de sus habitantes, potenciar los accesos para actividades culturales, apoyo a la industria artesanal y pequeño comercio, programas sociales y mejora de la actividad económica.

La inversión total prevista en el Centro Histórico hasta el año 2002 es de 52.489 millones de pesetas (315 millones de euros).

\section{SUMMARY}

Due to its large surface, over 320 hectares, the historical centre of Madrid included areas with a very high level of architectural, urban, social and environmental decay, there being greatly marginalised districts which shelter immigrant population.

In view of this on-going urban decay of the historical centre, the three administrations (state, regional and local) entered several agreements for the Rehabilitation of the Residential and Urban Heritage, which, together with the Cohesion Funds and the URBAN projects for Cinema and Theatre Itineraries, both financed by the European Union, intend to regenerate the urban base. Coordination and management are in the hands of the Municipal Housing Corporation.

The formula selected to intervene in the historical centre has been the declaration of Preferential Rehabilitation Areas, which focuses on the squares or main streets in cach district.

Another formula to intervene has been the rehabilitation of streets within the Cinema and Theatre Itineraries under the $U R B A N$ projects, the base of which is connected to the rehabilitation areas containing streets with prostitution and social marginalisation. The Cohesion Funds to upgrade the urban environment of Lavapies aim at adapting this district to the new needs of its population.

Interventions aim at infrastructure renovation, street design improvement, accessibility, environment upgrading. rehabilitation of housing blocks to improve the quality of life of their inhabitants, developing accesses for cultural activities, support for the arts and crafts industry and small enterprises, social programmes and economic activity improvement.

Total investment in the Historical Centre amounts to 52,489 million pesetas (315 million euros) up to the year 2002. 


\section{SITUACIÓNANTERIORALAREHABILITACIÓN}

El Centro Histórico de Madrid, uno de los más grandes de Europa con cerca de 400 hectáreas, presentaba, junto a zonas monumentales y emblemáticas, otras de alto nivel de deterioro social, arquitectónico y urbanístico, escasamente habitadas por sectores de población envejecida, inmigrante y marginada que habia provocado una gran pérdida, entre otras, de las actividades económicas tradicionales.

\section{PROCESODE LA REHABILITACIÓN}

Ante esta alarmante situación las tres Administraciones, Local, Regional y Estatal (Ayuntamiento, Comunidad de Madrid y Ministerio de Fomento), respectivamente, firmaron los "Convenios para la Rehabilitación del Patrimonio Edificado Residencial y Urbano", concentrando sus actuaciones en Áreas de Rehabilitación Preferente, es decir, en zonas de deterioro homogéneas.

Cabe destacar que con el ejemplo de las Administraciones se ha impulsado la acción de la iniciativa privada, que se ha sumado desde el comienzo al proceso de recuperación del Centro Histórico.

Paralelamente se han firmado Convenios de colaboración con las Compañias de Servicios y Colegios Profesionales contando con el apoyo de las Asociaciones de Vecinos y Comerciantes.

Como complemento a la actuación en Áreas de Rehabilitación Preferente se han conseguido Fondos de la Unión Europea y de Entidades Financieras.

La gestión de estas iniciativas es llevada a cabo por la Empresa Municipal de la Vivienda de Madrid, S.A. (EM V) en colaboración con las Tenencias de Alcaldia, Áreas y Juntas Municipales de Distrito y la Comunidad de Madrid(Dirección General de Arquitectura y Vivienda de la Consejería de Obras Públicas, Urbanismo y Transportes) así como de la Dirección General de la Vivienda, la Arquitectura y el Urbanismo del Ministerio de Fomento.

Los programas de intervención son los siguientes:

\section{Rehabilitación privada}

Los propietarios o usuarios de las viviendas rehabilitan sus edificios acogiéndose a las Ayudas de las tres Administraciones. Consisten en subvenciones a fondo perdido que pueden llegar hasta el $60 \%$ del presupuesto. El objetivo es el logro de las debidas condiciones de habitabilidad de las viviendas.

Las obras se ejecutarán por fases, siendo las primeras, las imprescindibles para garantizar la seguridad del edificio.
Las actuaciones se han dividido en dos grupos:

- Obras de primer orden. Son las de cimentación, estructuras, saneamiento, cubierta y fachada.

- Obras o instalaciones, como los patios, accesos, instalación de ascensores, antenas colectivas, aislamientos, etc.

En el Barrio de Maravillas, dentro del Área I-Plaza del Dos de Mayo, existen numerosos edificios de corralas con un aseo por planta, careciendo de baño e, incluso, agua corriente en las viviendas. A partir de la declaración del Área, cerca del $60 \%$ de los inmuebles han sido rehabilitados y construidos baños dentro de las viviendas.

\section{Urbanización e infraestructuras}

Las Administraciones financian el $100 \%$ de las obras de plazas y calles, renovando las instalaciones y los pavimentos. La finalidad propuesta es la creación de espacios verdes, estanciales y de convivencia que mejoran las condiciones medioambientales, la seguridad ciudadana, el tráfico y la accesibilidad.

Las obras de urbanización realizadas en el Barrio de Maravillas (en el entomo a la plaza del Dos de Mayo) han consistido en la recuperación de los itinerarios tradicionales del barrio que se hallaban transformados por el tráfico de vehículos. Unos itinerarios de acceso a la plaza se han resuelto eliminando el aparcamiento y utilizando un trazado de coexistencia, sin bordillos, como las calles Daoiz y Velarde; en el otro itinerario, el más importante, desde la glorieta de Bilbao, calle de Manuela Malasaña y calle de Ruiz, ha sido potenciado el tránsito peatonal, con la creación de estancias peatonales, ensanches de aceras y eliminación de aparcamientos.

Otros objetivos fundamentales fueron: la accesibilidad urbana, al enrasar las calzadas y aceras, moderación del tráfico con adoquinado, plazas señalizadas, reducción visual del ancho de calzada al incorporar arbolado, señalización de pasos de peatones con adoquinado de coloración diferente y mejorar el medio ambiente urbano con la incorporación de arbolado de pequeño porte, con riego por goteo y especies de distinta floración, perenne y caduca.

El entorno de la plaza Mayor, Área II de Rehabilitación Preferente, ha tenido un condicionante en los accesos al aparcamiento existente bajo la plaza, lo cual ha obligado a tener que realizar una actuación de transformación global de los pasos para reducir el impacto visual de las entradas en este marco histórico.

El paso de la calle Felipe III se ha reducido en su ancho, ataluzando los muros en su longitud, cubriendo parte del paso, aumentando la pendiente, también han sido planta- 
dos árboles de pequeño porte y tratado los pavimentos, con adoquín en calzada y granito en aceras. Las barandillas, colocadas con acero inoxidable y vidrio laminar.

El paso de salida de la calle de la Sal y su carril de circulación a la calle Postas y San Cristóbal ha sido eliminado, recuperando el paseo peatonal que une la Puerta del Sol con la plaza Mayor.

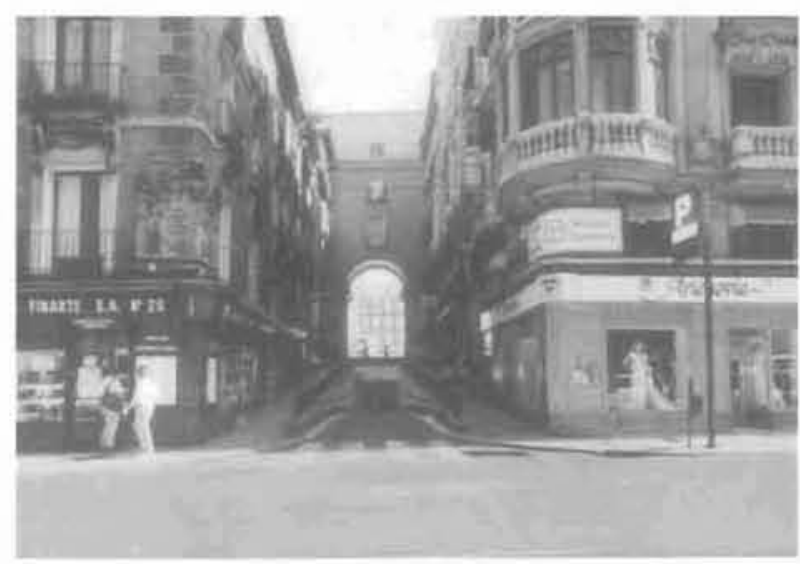

Calle Mayor, 25. Acceso a plaza Mayor y al aparcamiento subterráneo
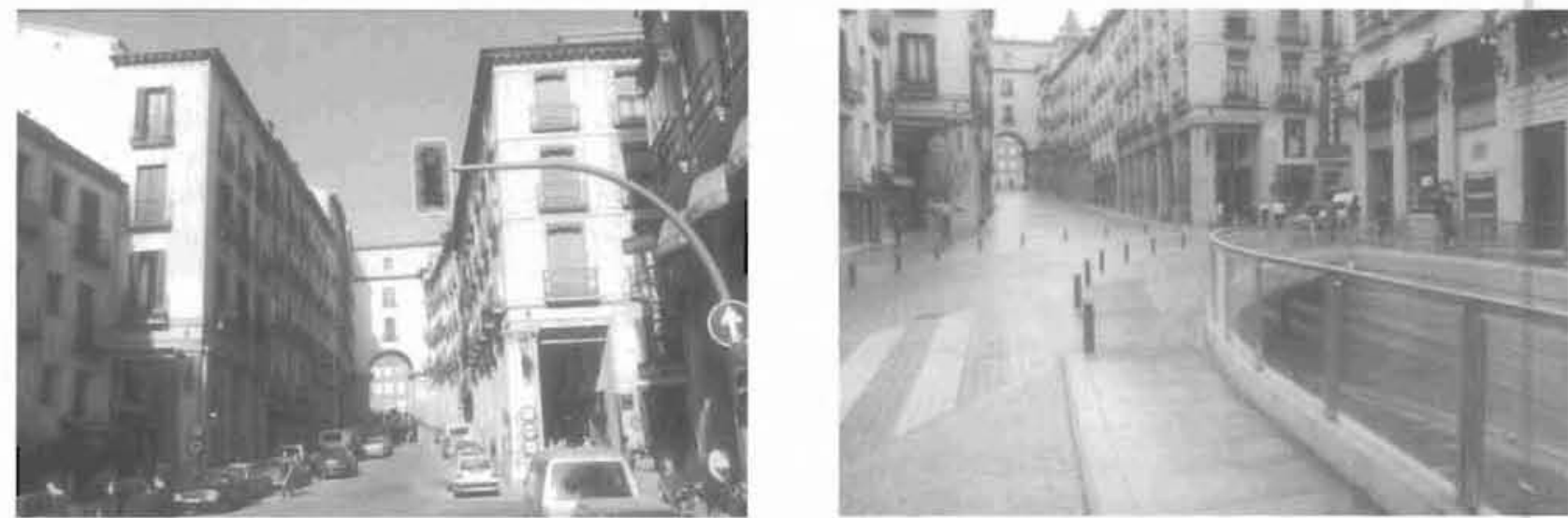

Calle Toledo. Acceso a plaza Mayor y subterráneo bajo la misma.

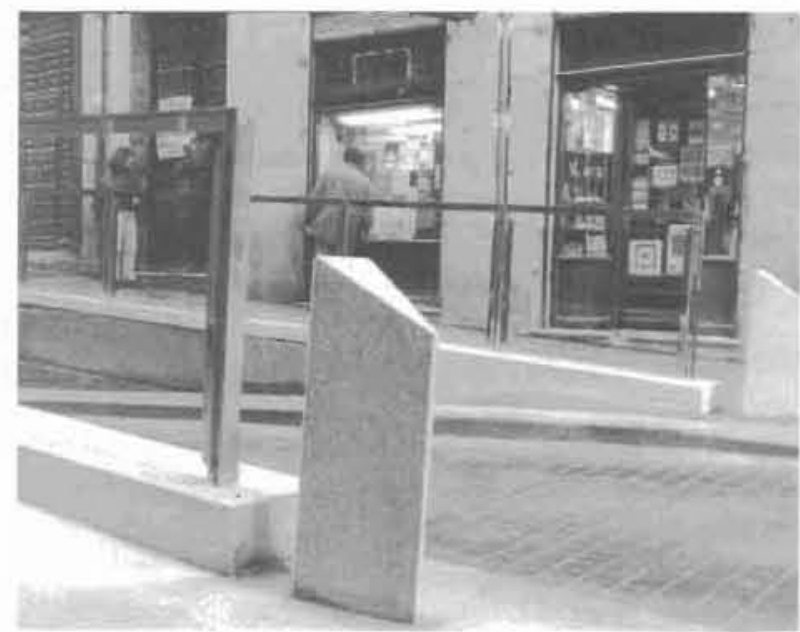

Plaza Mayory su entorno. Detalle de bolardos de proteccion y barandillas de la entrada al aparcamiento por Felipe III.
Ha sido renovado el acceso peatonal de la calle Zaragoza con los mismos materiales utilizados en la calle Felipe III.

La calle de Toledo ha sido la que ha tenido una intervención mayor al eliminar el tráfico de vehículos en el tramo porticado diseñado por Juan de Villanueva, recuperando su tradicional conexión peatonal con Puerta Cerrada.

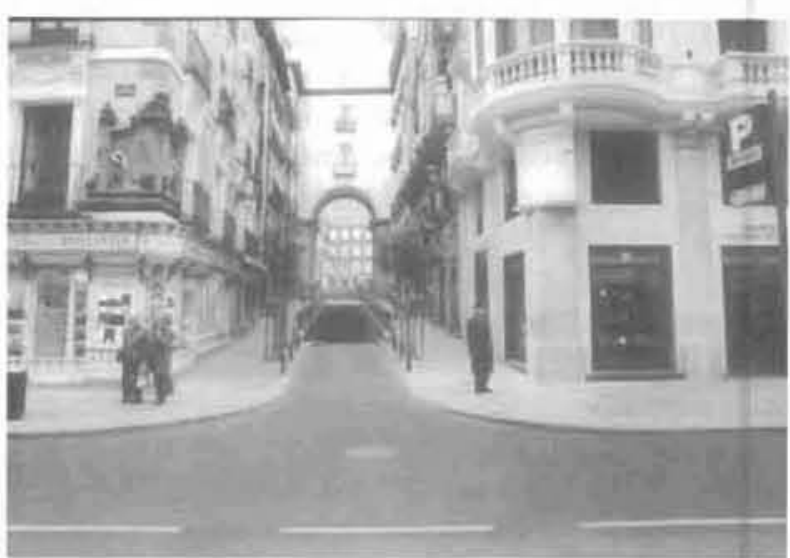


Al trasladar la entrada de vehículos a la calle Concepción Jerónima también se ha dado salida a los vehículos del aparcamiento hacia el sur $y$, a su vez, ha permitido cerrar la salida de la calle de la Sal.

El tratamiento dado a esta calle ha sido similar a las otras del Área, con pavimento de granito, barandillas de acero y vidrio, muros de GRC con listelos de cobre y adoquin artificial en calzadas.

En este Área también se han remodelado las calles de Cuchilleros y Cava de San Miguel, donde se han introducido dos conductos de $90 \mathrm{~cm}$ de diámetro para suministro de agua, al tener que eliminar el tramo de la calle Toledo al hacer el subterráneo.

También han sido urbanizadas las calles de Zaragoza, Fresa, Imperial, Siete de Julio, Marqués Viudo de Pontejos y los soportales de Gerona y plazas de la Provincia y de Santa Cruz. Con estas intervenciones han sido recuperadas todas las calles del entorno de la plaza Mayor.

En todas las obras de urbanización de las Áreas han sido renovadas las instalaciones urbanas (agua, electricidad, alumbrado público, telefónica, gas y colocados conductos para fibra óptica)

\section{Programas de adecuación arquitectónica}

Las obras de algunas calles, plazas más representativas y sus fachadas son realizadas por las Administraciones al $100 \%$, sirviendo de atractivo a la iniciativa privada, que completará la rehabilitación del interior de los edificios con las ayudas, mencionadas anteriormente, de hasta el $60 \%$ del presupuesto.

Los Programas resultan ser una conjunción de actuaciones, encaminadas a la recuperación de espacios singulares de cada Área, como motor de la tarea rehabilitadora.

La intervención pública en fachadas ha consistido, básicamente, en la recuperación de revocos tradicionales, mediante una investigación previa en archivos y en el propio edificio, restauración de carpinterías, cerrajerías y molduraciones o fingidos en recercado de huecos, esgrafiados, etc.

Los Programas realizados han sido: la plaza del Dos de Mayo, que incluye su urbanización, las calles del entorno de la plaza de la Paja, y el pasaje Arco del Triunfo.

\section{Programas sociales}

La Empresa Municipal de la Vivienda adquiere edificios destinados al alquiler, efectúa realojos y subvenciona, a través de los Convenios, hasta el $100 \%$ del presupuesto en casos extremos de insolvencia, consiguiendo el bien-
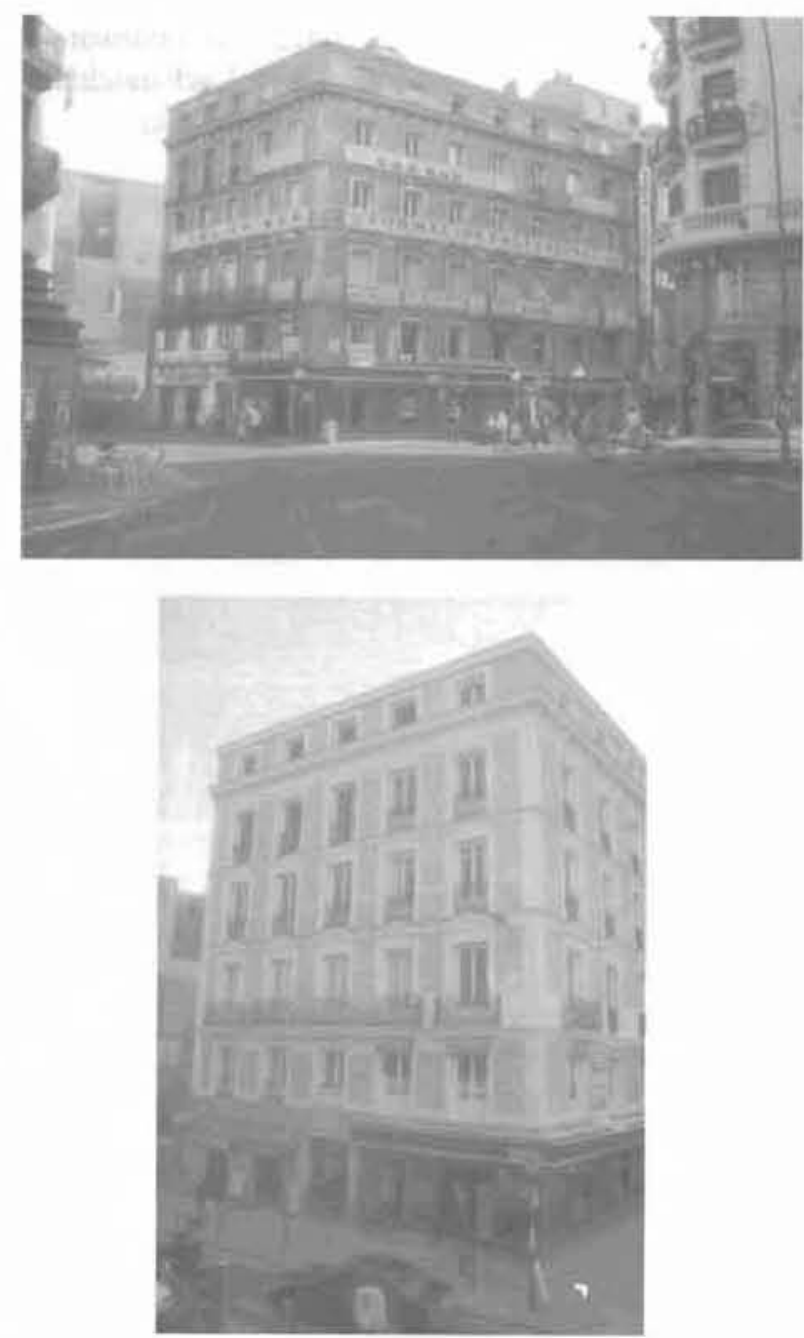

Calle Fuencarral, 105. Rehabilitaciỏn de fachadas

estar social deseable. Se completan estas actuaciones con programas específicos como son los de ludoteca y mujer marginada.

\section{Actuaciones monumentales}

Como complemento de la actividad de rehabilitación, la Empresa Municipal de la Vivienda realiza actuaciones en edificios y conjuntos singulares de carácter monumental, valorando sus condiciones histórico-artisticas y su calidad arquitectónica y emblemática.

\section{SITUACIÓNACTUAL}

Estando próxima la finalización de los primeros Convenios: Área plaza del Dos de Mayo, Área plaza Mayor y Área de las plazas de la Paja, de los Carros, del Alamillo y de la Cruz Verde, se puede decir que los resultados están siendo óptimos, con la finalización de los Programas de Adecuación Arquitectónica y prácticamente de las obras de Infraestructuras, habiéndose recibido más de un $60 \%$ 

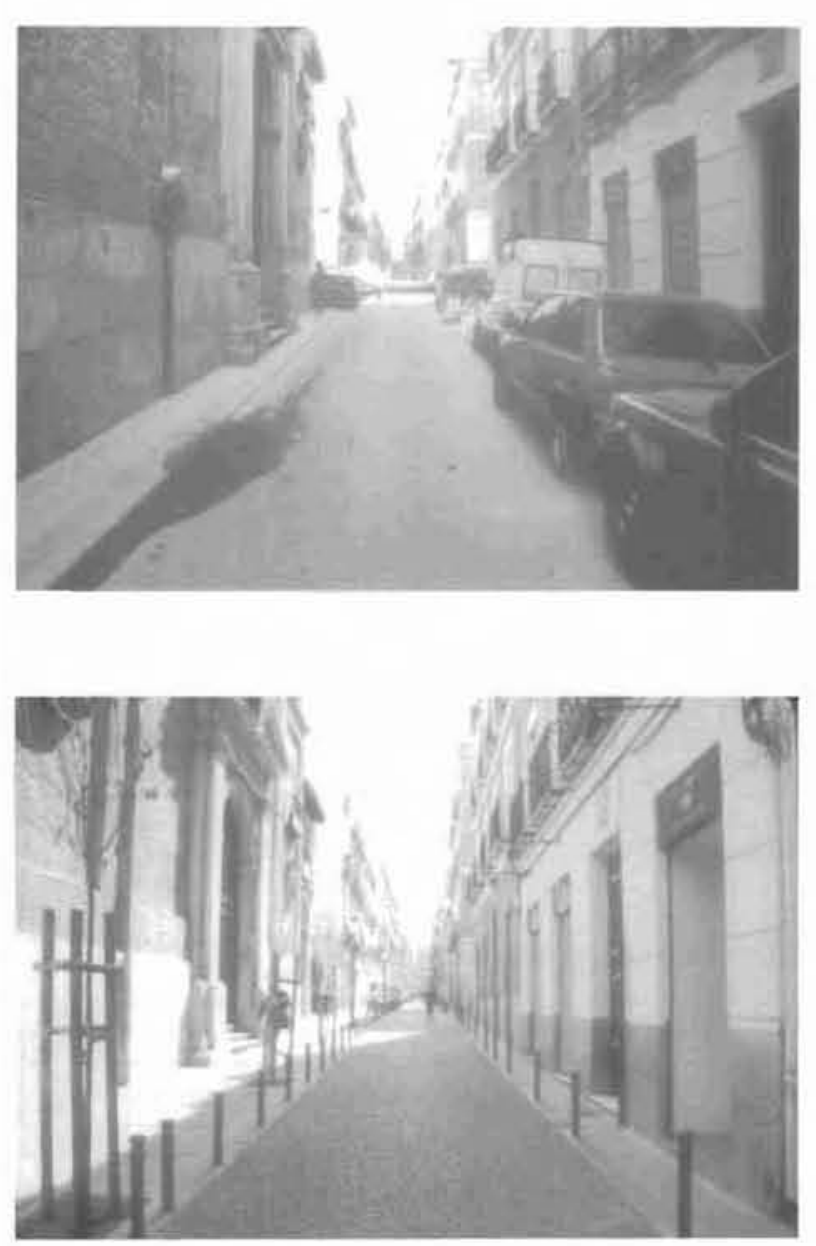

Rehabilitacion d: la calle de la Palma.

de solicitudes de Ayudas para la rehabilitación de las viviendas, logrando asi su confort y una mayor conciencia social de sus habitantes.

Estos resultados, motivaron la declaración de posteriores Áreas: Eje de la calle Mayor, Eje de la calle Fuencarral y de Lavapiés, actualmente en curso.

Asimismo, con la incorporación de los Fondos Europeos FEDER, se está logrando la Recuperación de itinerarios históricos y culturales, desarrollados con el Proyecto URBAN de "Itinerarios de cines y teatros". Los también Fondos Europeos de Cohesión contribuyen a la mejora, a través del "Proyecto Medioambiental del Sector 1 de Lavapiés", de este Área de Rehabilitación Preferente.

Otros logros conseguidos son los del fomento del turismo, el aumento de la economía del pequeño comercio y la recuperación de oficios y artesanias tradicionales.

También las entidades financieras (Fundación Caja Madrid e Ibercaja) se sumaron al proceso, aportando importantes

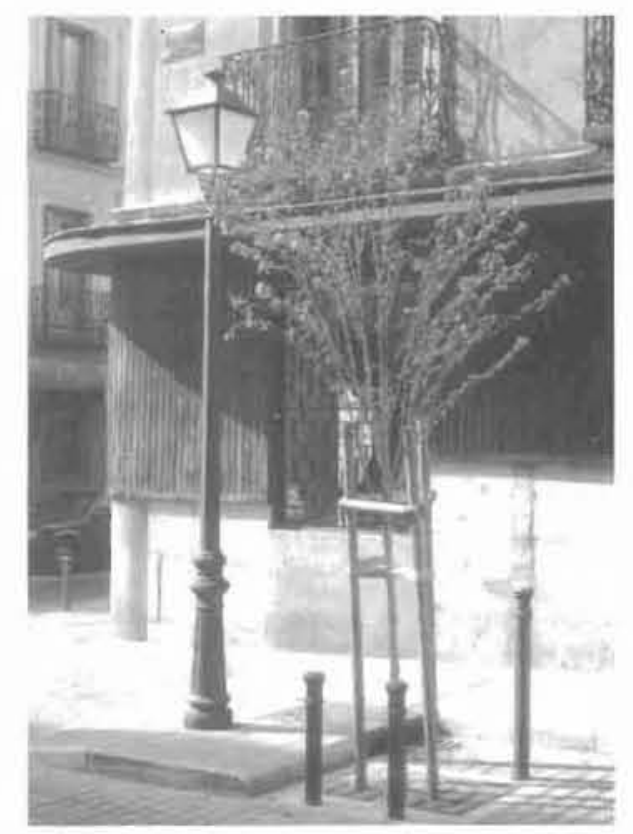

Plaza del Dos de Mayo, mobiliario urbano. Calle Montelein.

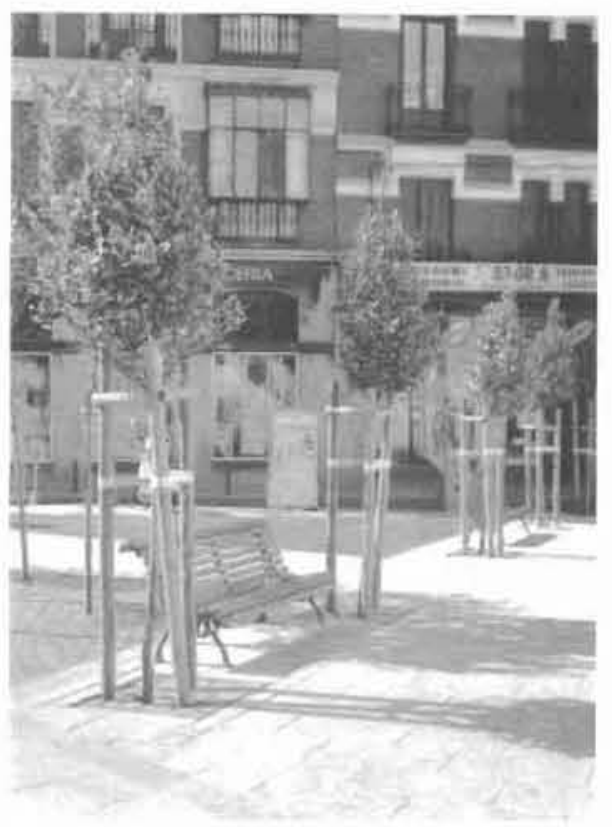

Plaza del Dos de Mayo, estancias peatonales. Calle Fuencarral con Divino Pastor.

cantidades. Otros Bancos y Cajas nacionales han firmado Convenios con condiciones ventajosas para la rehabilitación de viviendas.

En el caso de las actuaciones monumentales singulares, y porserla más reciente, nos referimos aquía la rehabilitación del Salón Real en la Casa de la Panadería, situado en la plaza Mayor, donde las obras, financiadas por la Fundación Caja Madrid, han conseguido la recuperación de un importante marco para acontecimientos públicos oficiales de gran relieve cultural, en semejanza a su histórico uso original. 


\section{UNAINICIATIVAASEGUIR}

La iniciativa madrileña de actuación en Áreas de Rehabilitación Preferente ha abierto un camino seguido por otras ciudades, por lo que nos sentimos satisfechos de haber contribuido a impulsar los trabajos de otros Ayuntamientos españoles, europeos e iberoamericanos que nos han visitado en los últimos años.

\section{DISTRIBUCION DE LAS INVERSIONES}

CONVENIOS AYUNTAMIENTO - COMUNIDAD DE MADRID - MINISTERIO DE FOMENTO

\begin{tabular}{|c|c|c|c|c|c|c|c|}
\hline \multicolumn{6}{|c|}{ INVERSIÓN CONVENIO } & \multirow{2}{*}{$\begin{array}{l}\text { FUERA } \\
\text { CONVENIO } \\
\text { U.EUROPEA/ } \\
\text { AYTO. }\end{array}$} & \multirow{2}{*}{$\begin{array}{c}\text { TOTAL } \\
\text { INVERSIÓN } \\
\text { ÁREA }\end{array}$} \\
\hline ÁREA & $\begin{array}{c}N^{\circ} . \\
\text { VIVIENDAS }\end{array}$ & $\begin{array}{c}N^{\circ} . \\
\text { EDIFICIOS }\end{array}$ & $\begin{array}{l}\text { INVERSION } \\
\text { PUBLICA }\end{array}$ & $\begin{array}{l}\text { INVERSIÓN } \\
\text { PRIVADA }\end{array}$ & $\begin{array}{c}\text { TOTAL } \\
\text { INVERSIÓN }\end{array}$ & & \\
\hline Plz. Dos de Mayo & 6.750 & 601 & 4.523 & 2.385 & 6.908 & . & 6.908 \\
\hline Plz. Mayor-Arco Triunto & 1.318 & 137 & 2.470 & 945 & 3.415 & . & 3.415 \\
\hline $\begin{array}{l}\text { Plz.Paja, Carros, Alamillo } \\
\text { y Cruz Verde }\end{array}$ & 927 & 96 & 834 & 534 & 1.338 & - & 1.338 \\
\hline Eje calle Fuencarral & 3.213 & 267 & 1.851 & 436 & 2.287 & - & 2.287 \\
\hline Eje calle Mayor & 1.891 & 180 & 1.784 & 396 & 2.180 & . & 2.180 \\
\hline Lavapiés & 11.788 & 756 & 6.572 & 2.558 & 9.130 & $8.466,6$ & $17.596,6$ \\
\hline TOTAL & 25.887 & 2.037 & 18.034 & 7.254 & 25.258 & $8.466,6$ & $33.724,6$ \\
\hline
\end{tabular}

CONVENIOS AYUNTAMIENTO - FONDOS EUROPEOS - ENTIDADES FINANCIERAS

\begin{tabular}{|l|r|}
\hline AREA DE OBRAS E INFAAESTRUCTURAS (Monumentos, equipamientos, calles) & 13.500 \\
\hline PROYECTO URBAN - FONDOS EUROPEOS FEDER & 4.500 \\
\hline CONVENIO AYUNTAMIENTO - FUNDACION CAJA MADRID & 600 \\
\hline CONVENIO AYUNTAMIENTO - IBERCAJA & 35 \\
\hline TOTAL. & 18.635 \\
\hline
\end{tabular}

TOTAL INVERSIÓN CENTRO HISTORICO DE MADRID $52.359,6$ millones de pesetas

TOTAL INVERSION CENTRO HISTORICO DE MADRID 313.530 .530 EUROS 